\title{
Correction to: MiDBench: Multimodel Industrial Big Data Benchmark
}

Yijian Cheng, Mengqian Cheng, Hao Ge, Yuhe Guo, Yuanzhe Hao, Xiaoguang Sun, Xiongpai Qin, Wei Lu, Yueguo Chen, and Xiaoyong Du

\section{Correction to:}

\section{Chapter "MiDBench: Multimodel Industrial Big Data} Benchmark" in: C. Zheng and J. Zhan (Eds.):

Benchmarking, Measuring, and Optimizing, LNCS 11459, https://doi.org/10.1007/978-3-030-32813-9_15

In the version of this paper that was originally published, reference 3 linked to the wrong website. This has been corrected. 